\title{
ON THE EDGE SET OF GRAPHS OF LATTICE PATHS
}

\author{
STEVEN KLEE, LARA PUDWELL, and RICK GILLMAN
}

Received 4 June 2003

\begin{abstract}
This note explores a new family of graphs defined on the set of paths of the $m \times n$ lattice. We let each of the paths of the lattice be represented by a vertex, and connect two vertices by an edge if the corresponding paths share more than $k$ steps, where $k$ is a fixed parameter $0=k=m+n$. Each such graph is denoted by $G(m, n, k)$. Two large complete subgraphs of $G(m, n, k)$ are described for all values of $m, n$, and $k$. The size of the edge set is determined for $n=2$, and a complicated recursive formula is given for the size of the edge set when $k=1$.
\end{abstract}

2000 Mathematics Subject Classification: 05C75, 05A99.

1. Introduction. A classic combinatorial problem, presented in nearly every introductory text, is enumerating the number of distinct paths on an $m \times n$ rectangular lattice. For the purposes of this note, we let $m$ denote the number of rows and $n$ denote the number of columns of rectangular cells in the lattice.

This problem is often modelled as walking along a rectangular grid of square city blocks. Following Gillman [2], we say two paths are essentially the same, or $(k+1)$ equivalent, if they share more than $k$ steps (or, conversely, they are $k$-distinct if they share no more than $k$ steps). Paths are denoted as a sequence of $m$ North steps and $n$ East steps on the lattice ( $\mathrm{N}$ and $\mathrm{E}$, respectively). Also, without loss of generality, allow $m \geq n$ due to the symmetry of the lattice. For simplicity, we let $C$ denote $\left(\begin{array}{c}m+n \\ n\end{array}\right)$ and will let $\left\{P_{1}, P_{2}, \ldots, P_{C}\right\}$ denote the set of all paths on the $m \times n$ lattice, with paths listed in reverse lexicographic order.

The set of all paths on the $m \times n$ lattice, denoted as $L(m, n)$, can be viewed as the vertices of a graph. The edges of the graph will connect those paths that are $(k+1)$ equivalent. This graph is denoted as $G(m, n, k)$, and its edge set is $\mathrm{E}(m, n, k)$.

2. Barrier paths. This section describes two complete subgraphs of $G(m, n, k)$.

DEFINITION 2.1. In $L(m, n)$, the paths of the forms $\mathrm{N}^{k+1} \mathrm{E}^{n} \mathrm{~N}^{m-(k+1)}$ and $\mathrm{E}^{k+1} \mathrm{~N}^{m}$ $\mathrm{E}^{n-(k+1)}$ are called barrier paths and denoted as $P_{y_{k}}$ and $P_{x_{k}}$, respectively, when the paths of the $m \times n$ lattice are listed in reverse lexicographic order.

RESULT 2.2. The index $y_{k}$ is found by

$$
y_{k}=\left(\begin{array}{c}
m+n-k-1 \\
n
\end{array}\right)
$$


Proof. $\quad P_{y_{k}}$ is of the form $\mathrm{N}^{k+1} \mathrm{E}^{n} \mathrm{~N}^{m-(k+1)}$ and encloses an $(m-(k+1)) \times n$ sublattice of $L(m, n)$. Since $P_{y_{k}}$ is the final path in this sublattice, $y_{k}=\left(\begin{array}{c}m+n-k-1 \\ n\end{array}\right)$.

RESULT 2.3. The index $x_{k}$ is found recursively by

$$
x_{k}=\left(\begin{array}{c}
m-k+n-1 \\
m-1
\end{array}\right)+x_{k-1} \quad \text { with } x_{0}=\left(\begin{array}{c}
m+n-1 \\
m-1
\end{array}\right)+1
$$

Proof. $P_{x_{0}}$ immediately follows the path $P_{y_{0}}$. Since $y_{0}=\left(\begin{array}{c}m+n-1 \\ n\end{array}\right)$ from Result 2.2, it is easy to see that $x_{0}=\left(\begin{array}{c}m+n-1 \\ n\end{array}\right)+1$.

$P_{x_{k}}$ follows any path of the form $\mathrm{E}^{k} \mathrm{NE}^{n-k} \mathrm{~N}^{m-1}$. This means that $P_{x_{k-1}}$ and $P_{x_{k}-1}$ enclose an $(n-k) \times(m-1)$ sublattice of $L(m, n)$, and $x_{k}-x_{k-1}=\left(\begin{array}{c}m-k+n-1 \\ m-1\end{array}\right)$. Thus, $x_{k}=\left(\begin{array}{c}m-k+n-1 \\ m-1\end{array}\right)+x_{k-1}$.

Theorem 2.4. The sets $A=\left\{P_{1}, \ldots, P_{y_{k}}\right\}$ and $B=\left\{P_{x_{k}}, \ldots, P_{C}\right\}$ induce complete subgraphs of $G(m, n, k)$.

Proof. Any path in $A$ begins with $(k+1) \mathrm{N}$ steps. Thus, it follows that every path in $A$ is adjacent to every other path in the set. Therefore, $A$ forms a complete subgraph of $G(m, n, k)$.

Likewise, any path in $B$ begins with $(k+1)$ E steps. Thus, it follows that every path in $B$ is adjacent to every other path in the set. Therefore, $B$ forms a complete subgraph of $G(m, n, k)$.

It is important to note that neither of the induced subgraphs on $A$ or $B$ is necessarily maximal. Consider the paths $P=\mathrm{E}^{k}(\mathrm{NE})^{m-1} \mathrm{~N}$ and $Q=\mathrm{N}^{k}(\mathrm{EN})^{n-1} \mathrm{E}$. $P$ is adjacent to every path in $B$ and $Q$ is adjacent to every path in $A$, so neither subgraph is maximal.

3. Some special cases. Brewer et al. [1] determined the size of $G(m, 1, k)$ in the following theorem.

THEOREM 3.1. If $0 \leq k \leq m-1$,

$$
|\mathrm{E}(m, 1, k)|=\left(\begin{array}{c}
m-1 \\
2
\end{array}\right)-\left(\begin{array}{c}
k+2 \\
2
\end{array}\right)
$$

Since the size of $G(m, 1, k)$ has been determined, we turn our attention to the size of $G(m, 2, k)$ and begin by considering the extreme cases.

DEFINITION 3.2. Let $p(m, n, k)$ denote the number of pairs of paths in $L(m, n)$ that share exactly $k$ steps. For $k \geq 1$,

$$
p(m, n, k)=|\mathrm{E}(m, n, k-1)|-|\mathrm{E}(m, n, k)|
$$

Let $\mathrm{E}_{X}^{\prime}(Y)$ denote the set of pairs of paths, one in set $X$ and the other in set $Y$, that do not share any steps. 
THEOREM 3.3. If $m \geq 2$,

$$
|\mathrm{E}(m, 2,0)|=3\left(\begin{array}{c}
m+3 \\
4
\end{array}\right)-\left(\begin{array}{c}
m+1 \\
2
\end{array}\right)-2(m-1) .
$$

Proof. Since the indices of the barrier paths are $y_{0}=\left(\begin{array}{c}m_{2}^{+1} \\ 2\end{array}\right)$ and $x_{0}=y_{0}+1=$ $\left(\begin{array}{c}m_{2}^{+1} \\ 2\end{array}\right)+1$, let $A=\left\{P_{1}, \ldots, P_{y_{0}}\right\}$ and $B=\left\{P_{x_{0}}, \ldots, P_{C}\right\}$.

There are $\left(\begin{array}{c}C \\ 2\end{array}\right)$ possible edges in $G(m, n, k)$. Since $p(m, 2,0)=\left|\mathrm{E}_{A}^{\prime}(A)\right|+\left|\mathrm{E}_{B}^{\prime}(B)\right|+$ $\left|\mathrm{E}_{A}^{\prime}(B)\right|$, that is, the total number of pairs of paths in $L(m, 2)$ that are disjoint, then $|\mathrm{E}(m, 2,0)|=\left(\begin{array}{c}C \\ 2\end{array}\right)-p(m, 2,0)$. It is clear that $\left|\mathrm{E}_{A}^{\prime}(A)\right|=\left|\mathrm{E}_{B}^{\prime}(B)\right|=0$ by Theorem 2.4 , so we are left to find $\left|\mathrm{E}_{A}^{\prime}(B)\right|$.

$P_{x_{0}}$ does not share any steps with any path of the form $\mathrm{N}^{i} \mathrm{E}^{2} \mathrm{~N}^{m-i}$ for $1 \leq i \leq$ $m-1$. Therefore, $\left|\mathrm{E}_{A}^{\prime}\left(\left\{P_{x_{0}}\right\}\right)\right|=m-1$. For $B^{*}=\left\{P_{x} \mid x_{0}+1 \leq x \leq C-1\right\}, P_{x}$ is of the form $\mathrm{EN}^{m-\left(x-x_{0}\right)} \mathrm{EN}^{x-x_{0}}$. Any $P_{x}$ does not share any steps with any path in the $\left(x-x_{0}\right) \times 1$ sublattice contained by $P_{1}$ and the path of the form $\mathrm{N}^{m-\left(x-x_{0}\right)} \mathrm{EN}^{x-x_{0}} \mathrm{E}$. Thus, $\left|\mathrm{E}_{A}^{\prime}\left(\left\{P_{x}\right\}\right)\right|=x-x_{0}+1$ and

$$
\left|\mathrm{E}_{A}^{\prime}\left(B^{*}\right)\right|=\sum_{x-x_{0}=1}^{m-1}\left(x-x_{0}+1\right)=\left(\begin{array}{c}
m \\
2
\end{array}\right)+m-1 .
$$

$P_{C}$ does not share any steps with any path of the form $\mathrm{N}^{i} \mathrm{EN}^{m-i} \mathrm{E}$ for $1 \leq i \leq m$. So $\left|\mathrm{E}_{A}^{\prime}\left(\left\{P_{C}\right\}\right)\right|=m$.

The above cases have accounted for every path in $B$, so

$$
\begin{aligned}
p(m, 2,0) & =\left|\mathrm{E}_{A}^{\prime}(B)\right| \\
& =m-1+\left(\begin{array}{c}
m \\
2
\end{array}\right)+m-1+m \\
& =\left(\begin{array}{c}
m+1 \\
2
\end{array}\right)+2(m-1) .
\end{aligned}
$$

Since $|\mathrm{E}(m, 2,0)|=\left(\begin{array}{l}c \\ 2\end{array}\right)-p(m, 2,0)$, through algebraic manipulation we have

$$
|\mathrm{E}(m, 2,0)|=3\left(\begin{array}{c}
m+3 \\
4
\end{array}\right)-\left(\begin{array}{c}
m+1 \\
2
\end{array}\right)-2(m-1) .
$$

LEMMA 3.4. There are

$$
\sum_{i=1}^{\left(\begin{array}{c}
m+2 \\
2
\end{array}\right)-1}(i)-|\mathrm{E}(m, 2,0)|
$$

pairs of disjoint paths in an $m \times 2$ lattice. 
Proof. There are $\left(\begin{array}{c}m+2 \\ 2\end{array}\right)$ vertices in $G(m, 2, k)$. The complete graph on these $\left(\begin{array}{c}m+2 \\ 2\end{array}\right)$ vertices has an edge for every pair of vertices, that is, for all pairs of paths in $L(m, 2)$. There are $\sum_{i=1}^{\left(\begin{array}{c}m+2 \\ 2\end{array}\right)-1} i$ edges in the complete graph on $\left(\begin{array}{c}m+2 \\ 2\end{array}\right)$ vertices. Further, $|\mathrm{E}(m, 2,0)|$ is the number of edges in $G(m, 2,0)$ representing pairs of paths that share at least 1 step, that is, not disjoint. Therefore, the number of pairs of disjoint paths in $L(m, 2)$ is the difference of these which are equal to

$$
\sum_{i=1}^{\left(\begin{array}{c}
m+2 \\
2
\end{array}\right)-1}(i)-|\mathrm{E}(m, 2,0)|
$$

THEOREM 3.5.

$$
|\mathrm{E}(m, 2,1)|=|\mathrm{E}(m, 2,0)|-2\left(\begin{array}{c}
m \\
2
\end{array}\right)-2(3 m-5) .
$$

Proof. Since $|\mathrm{E}(m, 2,0)|$ is the number of pairs of paths that share at least one step and $|\mathrm{E}(m, 2,1)|$ is the number of pairs of paths that share at least two steps in $L(m, 2)$, $|\mathrm{E}(m, 2,0)|-|\mathrm{E}(m, 2,1)|$ is the number of pairs of paths that share exactly one step. Consider all the ways two paths can share exactly one edge in $L(m, 2)$. Notice that any path must begin with either an $\mathrm{N}$ step or an E step and must end with either an $\mathrm{N}$ step or an E step. There is exactly one pair of disjoint paths that share either their first or last E step. Also, for every interior $\mathrm{N}$ step, of which there are $m-2$, there are two pairs of disjoint paths. Now we only have to find the number of pairs of disjoint paths in the $(m-1) \times 2$ sublattices consisting of paths that either begin or end with an $\mathrm{N}$ step. Combining this result with Lemma 3.4, we have

$$
|\mathrm{E}(m, 2,0)|-|\mathrm{E}(m, 2,1)|=2+2(m-2)+2\left[\sum_{i=1}^{\left(\begin{array}{c}
m+1 \\
2
\end{array}\right)-1}(i)-|\mathrm{E}(m-1,2,0)|\right] .
$$

Through algebraic manipulation, we arrive at our final result

$$
|\mathrm{E}(m, 2,1)|=|\mathrm{E}(m, 2,0)|-2\left(\begin{array}{c}
m \\
2
\end{array}\right)-2(3 m-5) .
$$

A path has a corner whenever an $\mathrm{N}$ step is followed immediately by an E step, or vice-versa. An $\mathrm{N}$-E transposition consists of reversing the $\mathrm{N}$ and $\mathrm{E}$ steps at a corner. For example, switching from ENNNEE to ENNENE is an N-E transposition, but switching from ENNNEE to EEENNN is not.

THEOREM 3.6. If $m \geq 2$,

$$
|\mathrm{E}(m, 2, m-1)|=2\left(\begin{array}{c}
m+1 \\
2
\end{array}\right)
$$

Proof. Two vertices in $G(m, 2, m-1)$ share an edge if and only if their corresponding paths in $L(m, 2)$ differ by exactly one N-E transposition. We will count all such pairs of equivalent paths. 
CASE 1 ( $P=\mathrm{EEN}^{m}$ or $\left.P=\mathrm{N}^{m} \mathrm{EE}\right)$. Each of these paths has exactly one corner at which to make an N-E transposition. Therefore, these paths yield two pairs of equivalent paths.

CASE $2\left(P=\mathrm{N}^{i} \mathrm{EEN}^{m-i}, 0<i<m\right.$, or $\left.P=\mathrm{EN}^{m} \mathrm{E}\right)$. Each of these paths has exactly two corners at which to make an N-E transposition. Further, notice that there are $m-1$ choices for $i$ and only one path of the form $\mathrm{EN}^{m} \mathrm{E}$, yielding $2 m$ new pairs of equivalent paths.

CASE $3\left(P=\mathrm{EN}^{i} \mathrm{EN}^{m-i}\right.$ or $\left.P=\mathrm{N}^{i} \mathrm{EN}^{m-i} \mathrm{E}, 0<i<m\right)$. Each of these paths has exactly three corners at which to make an N-E transposition. Since there are $2(m-1)$ paths of this form, there are $6(m-1)$ new pairs of equivalent paths.

CASE 4. Consider the remaining paths of the form $P=\mathrm{N}^{i} \mathrm{EN}^{j} \mathrm{EN}^{m-i-j}$, for $i, j>0$ and $i+j<m$. Each of these paths has exactly four corners at which to make an N-E transposition. Further, since there are $\left(\mathrm{m}_{2}^{+2}\right)-2(m-1)-m-2$ paths of this form, we have $4\left(\left(\begin{array}{c}m+2 \\ 2\end{array}\right)-2(m-1)-m-2\right)$ new pairs of equivalent paths.

Thus, we have accounted for every path in $L(m, 2)$ in one of the cases above, and for each path, we have counted all its equivalent paths. However, each pair of paths has been counted exactly twice, one time for each path in the pairing. Therefore, the size of $G(m, 2, m-1)$ is exactly half the number of pairs of equivalent paths counted above, that is,

$$
\begin{aligned}
2|\mathrm{E}(m, 2, m-1)| & =2+2 m+6(m-1)+4\left(\left(\begin{array}{c}
m+2 \\
2
\end{array}\right)-2(m-1)-m-2\right) \\
|\mathrm{E}(m, 2, m-1)| & =1+m+3(m-1)+2\left(\left(\begin{array}{c}
m+2 \\
2
\end{array}\right)-2(m-1)-m-2\right) \\
& =2\left(\begin{array}{c}
m+1 \\
2
\end{array}\right) .
\end{aligned}
$$

4. The size of $G(m, 2, k)$. Now we are ready to develop a formula for the number of edges in $G(m, 2, k)$ for all values of $k$. We begin with the following definition.

DEFINITION 4.1. The number of pairs of paths, $P_{1}$ and $P_{2}$, in $L(m, n)$ that share exactly $k$ steps given $P_{1}$ begins with an $\mathrm{N}$ step and $P_{2}$ begins with an E step is found by the function $g(m, n, k)$.

This definition gives us the following relationship.

LEMMA 4.2.

$$
p(m, 2, k)=p(m-1,2, k-1)+p(m, 1, k-1)+g(m, 2, k) .
$$

Proof. $p(m-1,2, k-1)$ is the number of pairs of paths that share exactly $k$ steps given both paths begin with an $\mathrm{N}$ step, $p(m, 1, k-1)$ is the number of pairs of paths that share exactly $k$ steps given both paths begin with an E step, and, by definition, $g(m, 2, k)$ is the number of pairs of paths that share exactly $k$ steps given one path begins with an $\mathrm{N}$ step and the other begins with an E step. Thus, all pairs of paths have been accounted for. 
LEMMA 4.3.

$$
g(m, 2, k)=m+(2 m-1)(m-k-1)-3\left(\begin{array}{c}
m-k-1 \\
2
\end{array}\right) .
$$

Proof. Begin by noting that

$$
g(m, 2, k)=g(m-1,2, k-1)+2(m-k)-3 .
$$

$g(m-1,2, k-1)$ is the number of pairs of paths sharing exactly $k-1$ steps, with one path beginning with an $\mathrm{N}$ step and the other beginning with an E step, but both paths ending with an $\mathrm{N}$ step. There are $(m-k-2)$ ways for two paths to share exactly $k \mathrm{~N}$ steps on the interior of the lattice, and each of these sets of $\mathrm{N}$ steps results in two pairs of paths that share exactly $k \mathrm{~N}$ steps and are disjoint elsewhere. Also, there is one pair of paths that share exactly $k$ steps such that they share a final E step. This accounts for all possible paths in $g(m, 2, k)$ and verifies that

$$
g(m, 2, k)=g(m-1,2, k-1)+2(m-k)-3 .
$$

Also, from Lemma 4.2 and Theorems 3.3 and 3.5,

$$
\begin{aligned}
g(m, 2,1) & =p(m, 2,1)-p(m-1,2,0)-p(m, 1,0) \\
& =m^{2}+5 m-10-\frac{1}{2}\left((m-1)^{2}+5(m-1)-4\right)-1 \\
& =\frac{1}{2}\left(m^{2}+7 m-14\right) .
\end{aligned}
$$

Thus, by back substitution on $g(m, 2, k)-g(m, 2, k-1)$, we have

$$
g(m, 2, k)=m+(2 m-1)(m-k-1)-3\left(\begin{array}{c}
m-k-1 \\
2
\end{array}\right) .
$$

\section{THEOREM 4.4.}

$$
p(m, 2, k)=\frac{k+1}{2}\left(m^{2}+5 m-(k+1)(k+4)\right) .
$$

Proof. We will proceed by induction. $p(m, 2,1)=m^{2}+5 m-10$ is a direct result of Theorem 3.5. Now assume $p(m, 2, i-1)=(i / 2)\left(m^{2}+5 m-(i)(i+3)\right)$. From Lemma 4.2,

$$
\begin{aligned}
p(m, 2, i) & =p(m-1,2, i-1)+i+g(m, 2, i) \\
& =\frac{i+1}{2}\left(m^{2}+5 m-(i+1)(i+4)\right) .
\end{aligned}
$$

Thus,

$$
p(m, 2, k)=\frac{k+1}{2}\left(m^{2}+5 m-(k+1)(k+4)\right) .
$$

This theorem leads directly to the result that we want, a general formula for $\mid \mathrm{E}(m, 2$, $k)$, which we now present as a corollary. 
COROLLARY 4.5. If $m \geq 2$ and $k<m$,

$|\mathrm{E}(m, 2, k)|=|\mathrm{E}(m, 2,0)|+3\left(\begin{array}{c}k+3 \\ 4\end{array}\right)+2 k(k+2)+\frac{2 k^{2}(k+1)-k(k+3) m(m+5)}{4}$.

Proof. From Theorem $4.4, p(m, 2, k)=((k+1) / 2)\left(m^{2}+5 m-(k+1)(k+4)\right)$. Thus,

$$
\begin{aligned}
|\mathrm{E}(m, 2, k)| & =|\mathrm{E}(m, 2,0)|+\sum_{i=1}^{k-1}\left(\frac{i+1}{2}\left(m^{2}+5 m-(i+1)(i+4)\right)\right) \\
& =|\mathrm{E}(m, 2,0)|+3\left(\begin{array}{c}
k+3 \\
4
\end{array}\right)+2 k(k+2)+\frac{2 k^{2}(k+1)-k(k+3) m(m+5)}{4} .
\end{aligned}
$$

5. A small generalization. The following theorem suggests the increasing complexity of the recursion formula as $n$ increases.

THEOREM 5.1.

$$
\begin{aligned}
& \left.|\mathrm{E}(m, n, 1)|=|\mathrm{E}(m, n, 0)|-2\left(\left(\begin{array}{c}
m+n-1 \\
m \\
2
\end{array}\right)\right)-|\mathrm{E}(m, n-1,0)|\right) \\
& \left.-2\left(\left(\begin{array}{c}
m+n-1 \\
n \\
2
\end{array}\right)\right)-|\mathrm{E}(m-1, n, 0)|\right) \\
& -\sum_{j=1}^{m-1} \sum_{i=1}^{n-2}\left(2\left(\left(\begin{array}{c}
i+j \\
i \\
2
\end{array}\right)-|\mathrm{E}(i, j, 0)|\right)\right. \\
& \left.\times\left(\left(\begin{array}{c}
m-j+n-i-1 \\
m-j \\
2
\end{array}\right)-|\mathrm{E}(m-j, n-i-1,0)|\right)\right) \\
& -\sum_{j=1}^{m-2} \sum_{i=1}^{n-1}\left(2\left(\left(\begin{array}{c}
i+j \\
i \\
2
\end{array}\right)\right)-|\mathrm{E}(i, j, 0)|\right) \\
& \left.\left.\times\left(\left(\begin{array}{c}
m-j+n-i-1 \\
n-i \\
2
\end{array}\right)\right)-|\mathrm{E}(m-j-1, n-i, 0)|\right)\right) .
\end{aligned}
$$

Proof. Notice that $|\mathrm{E}(m, n, 0)|-|\mathrm{E}(m, n, 1)|$ is the number of distinct paths in $L(m, n)$ that share exactly one edge. In $L(m, n)$, label an edge in terms of its distance from the lower left corner (i.e., set the origin as the lower left corner). First, we will count the pairs of paths that share exactly one E step.

(1) If two paths share an E step with label $(0, j)$ and $j>0$, they necessarily share at least one $\mathrm{N}$ step as well. Thus, there are no pairs of paths that share exactly one E step of this form. Similarly for sharing an E step with label $(n-1, j)$ and $j<m$ and for $(i, j)$ with $(i \neq 0$ or $n-1)$ and $(j=0$ or $m)$.

(2) Now, count all pairs of paths that share exactly the E step $(0,0)$. Clearly, this is the same as the number of disjoint paths in $L(m, n-1)$, that is, $\left(\begin{array}{c}\left(\begin{array}{c}m+n-1 \\ m\end{array}\right) \\ 2\end{array}\right)-|\mathrm{E}(m, n-1,0)|$. 
$\left(\begin{array}{c}m+n-1 \\ m\end{array}\right)$ is the number of paths in $L(m, n-1)$, and $\left(\begin{array}{c}\left(\begin{array}{c}m+n-1 \\ m\end{array}\right) \\ 2\end{array}\right)$ in $L(m, n-1) . \mid \mathrm{E}(m$, $n-1,0) \mid$ is the number of pairs of paths that share at least one step in $L(m, n-1)$. Thus, this difference is what we claim it is. Similarly for pairs of paths that share the E step $(n-1, m)$. Thus, there are $2\left(\left(\underset{2}{\left(\begin{array}{c}m+n-1 \\ m\end{array}\right)}\right)-|\mathrm{E}(m, n-1,0)|\right)$ that share exactly the E step $(0,0)$ or $(n-1, m)$.

(3) Now, count all pairs of paths that share exactly an E step $(i, j)$ that have not already been considered. There are $m-1$ choices for $i$ and $n-2$ choices for $j$. Then, for each $(i, j)$ pair, the number of pairs of paths that share this E step is twice the number of disjoint pairs of paths in $L(i, j)$ times the number of disjoint pairs of paths in $L(m-j, n-i-1)$, yielding

$$
\sum_{j=1}^{m-1} \sum_{i=1}^{n-2}\left(2\left(\left(\begin{array}{c}
\left(\begin{array}{c}
++j \\
i \\
2
\end{array}\right) \\
2
\end{array}\right)-|\mathrm{E}(i, j, 0)|\right)\left(\left(\begin{array}{c}
m-j+n-i-1 \\
m-j \\
2
\end{array}\right)-|\mathrm{E}(m-j, n-i-1,0)|\right)\right)
$$

additional pairs of paths that share exactly one more E step.

Thus, there are

$$
\begin{aligned}
& \left.2\left(\left(\begin{array}{c}
m+n-1 \\
m \\
2
\end{array}\right)\right)-|\mathrm{E}(m, n-1,0)|\right) \\
& \left.\left.\quad+2 \sum_{j=1}^{m-1} \sum_{i=1}^{n-2}\left(2\left(\left(\begin{array}{c}
i+j \\
i \\
2
\end{array}\right)\right)-|\mathrm{E}(i, j, 0)|\right)\left(\left(\begin{array}{c}
m-j+n-i-1 \\
m-j \\
2
\end{array}\right)\right)-|\mathrm{E}(m-j, n-i-1,0)|\right)\right)
\end{aligned}
$$

pairs of paths that share exactly one E step.

A similar argument shows that there are exactly

$$
\begin{aligned}
& 2\left(\left(\begin{array}{c}
m+n-1 \\
n \\
2
\end{array}\right)-|\mathrm{E}(m, n-1,0)|\right) \\
& \left.\left.+2 \sum_{j=1}^{m-2} \sum_{i=1}^{n-1}\left(2\left(\left(\begin{array}{c}
i+j \\
i \\
2
\end{array}\right)\right)-|\mathrm{E}(i, j, 0)|\right)\left(\left(\begin{array}{c}
m-j+n-i-1 \\
n-i \\
2
\end{array}\right)\right)-|\mathrm{E}(m-j-1, n-i, 0)|\right)\right)
\end{aligned}
$$

pairs of paths in $L(m, n)$ that share exactly one N step.

Combining the above two statements with some algebraic manipulation gives the stated theorem.

6. Conclusion. There are many other properties of these graphs that we could investigate, but our immediate attention will be on finding $|\mathrm{E}(m, n, k)|$ in general and in a closed form, and of the other invariants for this family of graphs. Of particular interest is the independence number, which was the original question posed in Gillman [2]. 


\section{REFERENCES}

[1] M. Brewer, A. Hughes, and L. Pudwell, Graphs of essentially equivalent lattice paths, Geombinatorics 13 (2003), no. 1, 5-9.

[2] R. Gillman, Enumerating and constructing essentially equivalent lattice paths, Geombinatorics 11 (2001), no. 2, 37-42.

Steven Klee: Department of Mathematics and Computer Science, Valparaiso University, Valparaiso, IN 46383, USA

E-mail address: steven.k1ee@va1po.edu

Lara Pudwell: Department of Mathematics, Rutgers University, Piscataway, NJ 08854, USA

E-mail address: 1pudwe11@math . rutgers . edu

Rick Gillman: Department of Mathematics and Computer Science, Valparaiso University, Valparaiso, IN 46383, USA

E-mail address: rick.gi11man@va7po.edu 


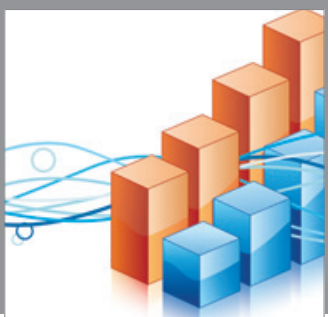

Advances in

Operations Research

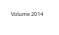

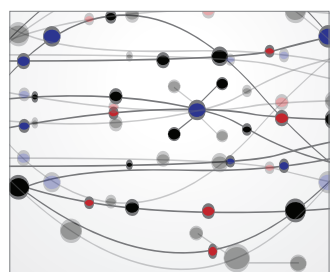

\section{The Scientific} World Journal
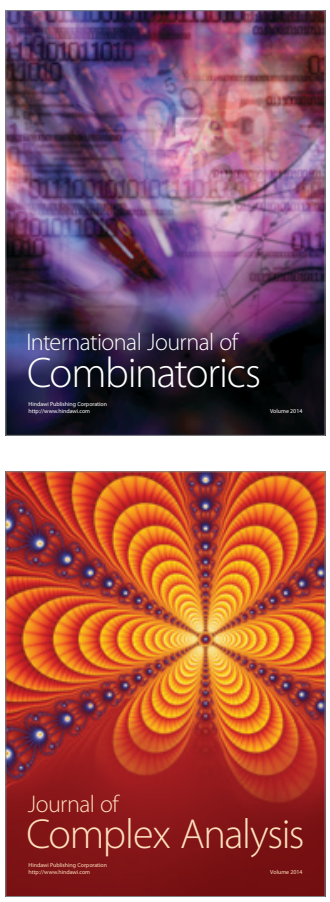

International Journal of

Mathematics and

Mathematical

Sciences
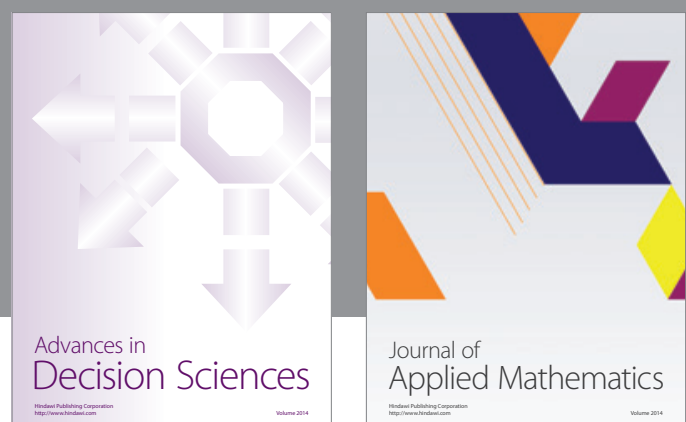

Journal of

Applied Mathematics
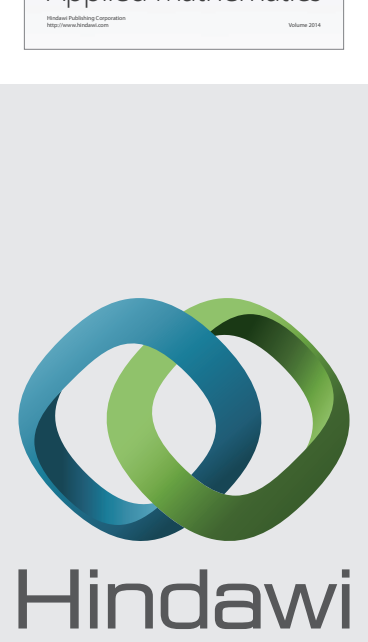

Submit your manuscripts at http://www.hindawi.com
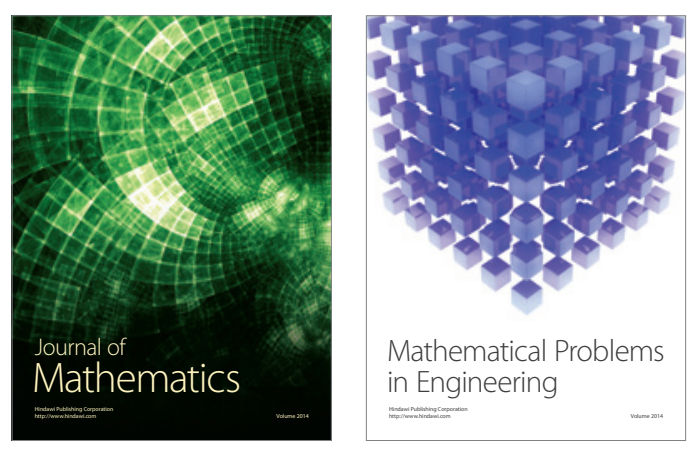

Mathematical Problems in Engineering
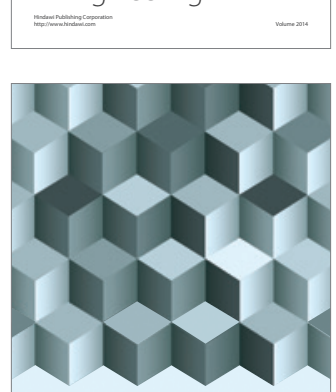

Journal of

Function Spaces
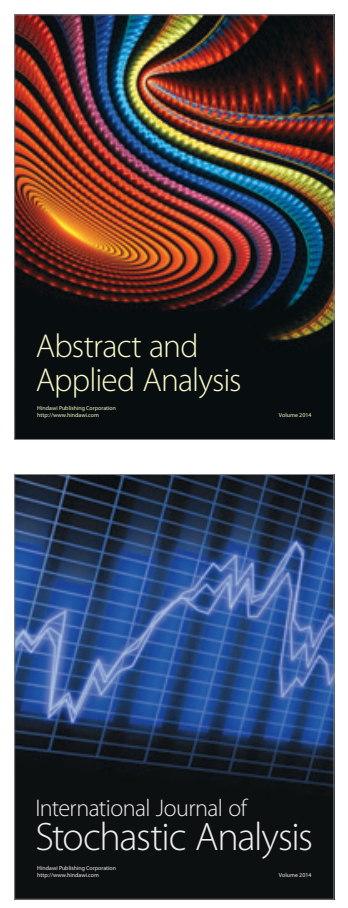

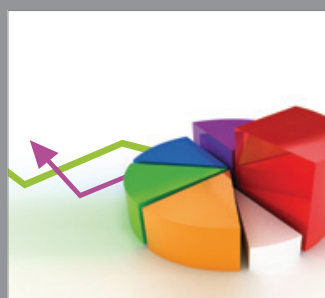

ournal of

Probability and Statistics

Promensencen
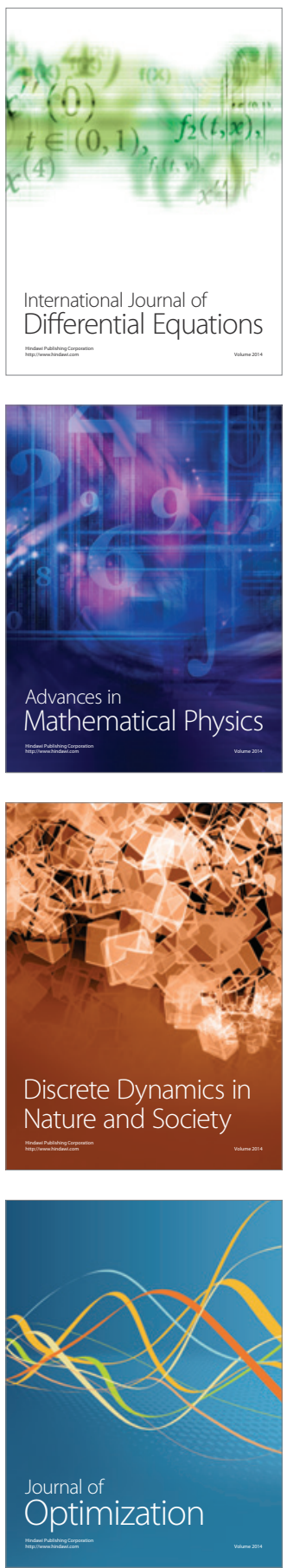